\title{
Reposicionamiento retrocapsular en un paciente con extrusión de prótesis peneana
}

\section{Retrocapsular repositioning in a patient with penile prosthesis extrusion}

\author{
Genaro Villela-Segura, ${ }^{1}$ Erick Alejandro Ramírez-Pérez, ${ }^{2}$ Damián López-Alvarado, ${ }^{3}$ Carlos Adrián Díaz de León- \\ Nevárez ${ }^{4}$
}

\begin{abstract}
Resumen
ANTECEDENTES: La colocación de prótesis peneanas representa la tercera línea de tratamiento en pacientes con disfunción eréctil. Una de las complicaciones más temidas es la extrusión distal de la prótesis, que ocurre cuando se debilita la túnica albugínea. CASO CLínICO: Paciente de 65 años de edad, con antecedente de colocación de prótesis peneana de 2 componentes; 8 años después tuvo disfunción mecánica, por lo que ameritó el recambio de la prótesis por una de 3 componentes. El paciente inició con dolor en la región distal-lateral derecha del cuerpo del pene. La resonancia magnética confirmó la extrusión de la prótesis sin perforación de la túnica albugínea. El procedimiento quirúrgico fue con la técnica de Mulcahy, con recambio protésico completo. El procedimiento se efectuó con corporotomía distal e incisión capsular transversal de la corpora en su posición dorsal, además de una nueva dilatación en la porción distal, y se colocó la prótesis en el nuevo trayecto (pseudocápsula). La prótesis peneana se activó a las 6 semanas posquirúrgicas, con evolución funcional satisfactoria y sin percepción de dolor por el paciente. La resonancia magnética de control mostró la prótesis en adecuada posición.

CONCLUSIÓN: La técnica de Mulcahy representa una opción efectiva, con alta tasa de éxito y baja de infección en pacientes con extrusión de prótesis en la porción lateral del pene.
\end{abstract}

PALABRAS CLAVE: Prótesis peneana; extrusión; técnica de Mulcahy.

\section{Abstract}

BACKGROUND: The placement of a penile prosthesis is third-line treatment in patients with erectile dysfunction. One of the most dreaded complications is distal extrusion of the prosthesis, which occurs when the tunica albuginea becomes weakened.

CLINICAL CASE: A 65-year-old man had a history of 2-piece penile prosthesis placement. Eight years later the prosthesis exhibited mechanical malfunction and was replaced with a 3-piece prosthesis. The patient's present illness began with pain in the right distal-lateral region of the body of the penis. Magnetic resonance imaging confirmed prosthesis extrusion, with no perforation of the tunica albuginea. Surgical procedure with the Mulcahy technique and replacement of the entire prosthesis was decided upon. The procedure was performed with distal corporotomy and a transversal capsular incision of the corpus in the dorsal position, as well as a new dilation in the distal portion. The prosthesis was placed in the new cavity (pseudocapsule). Six weeks after the surgery the penile prosthesis was activated, with satisfactory functional progression and no pain for the patient. Control magnetic resonance imaging showed adequate position of the prosthesis.

CONCLUSION: The Mulcahy technique is an effective option with a high success rate and low infection rate in patients with prosthesis extrusion in the lateral part of the penis. KEYWORDS: Penile Prosthesis; Extrusion; Mulcahy technique.
${ }^{1}$ Residente de Urología, Centro Médico Nacional 20 de Noviembre, ISSSTE, Ciudad de México.

${ }^{2}$ Centro de Uretra México, Hospital Ángeles Mocel, Ciudad de México.

${ }^{3}$ Centro de Uretra México, Hospital Licenciado Adolfo López Mateos, ISSSTE, Ciudad de México.

${ }^{4}$ Hospital General Santiago Ramón y Cajal, ISSSTE, Durango, México.

Recibido: octubre 2017

Aceptado: mayo 2018

Correspondencia

Genaro Villela Segura

gvillelauro@gmail.com

Este artículo debe citarse como Villela-Segura G, Ramírez-Pérez EA, López-Alvarado D, Díaz de León-Nevárez CA. Reposicionamiento retrocapsular en un paciente con extrusión de prótesis peneana. Rev Mex Urol. 2018 mayo-junio;78(3): 207-214. DOI:https://doi.org/10.24245/revmexurol.v78i3.1709 


\section{ANTECEDENTES}

Las indicaciones para la colocación de prótesis peneana incluyen: fracaso del tratamiento no quirúrgico, es decir, sujetos no aptos o con intolerancia al tratamiento médico o de segunda línea para la disfunción eréctil (inyección intracavernosa, tratamiento intrauretral o tópico), incluso que el paciente prefiera una opción permanente para este trastorno. ${ }^{1,2}$

En la actualidad existen dos tipos de prótesis peneanas: las inflables de 2 o 3 elementos y las maleables. Las prótesis inflables permiten erecciones más naturales, principalmente la de 3 elementos, que proporciona mayor rigidez y mejor flacidez; por su parte, las prótesis inflables de 2 elementos son efectivas en pacientes con riesgo en la colocación del reservorio.

Las prótesis semirrígidas se clasifican, a su vez, en maleables y mecánicas. Entre sus desventajas se encuentran que el pene permanece siempre en erección, si bien es posible orientarlo en tres posiciones diferentes: 1) recta-elevada para las relaciones sexuales, 2) hacia abajo para la micción, y 3) posición normal o doblado, con la finalidad de disimularlo con la ropa. ${ }^{3-5}$

La complicación más temida de la colocación de la prótesis peneana es la infección, cuya prevalencia varía de 1-2\%, debido a la modificación de las técnicas quirúrgicas y las nuevas presentaciones de prótesis recubiertas con antibiótico. Los casos de infección suelen registrarse en las primeras 8 semanas del posquirúrgico con: fiebre, dolor, eritema, fijación de la bomba escrotal a la piel, drenaje de pus y crepitación alrededor de la bomba o los cilindros. El porcentaje de infección después de la cirugía de rescate de la prótesis peneana es de $20 \%{ }^{6-8}$

Otra complicación reportada es la perforación uretral intraoperatoria, en la que se observan las puntas de los cilindros a través del meato uretral durante la cirugía. La extrusión de los cilindros de la prótesis peneana a través del meato uretral se observa frecuentemente en pacientes con prótesis maleables. Las perforaciones proximales y uretrales son complicaciones no infecciosas significativas que requieren reparación quirúrgica. ${ }^{9}$

De manera tardía puede observarse la extrusión de los cilindros de la prótesis peneana a través de la túnica albugínea, fuera de los cuerpos cavernosos sin perforar la uretra, incluso pueden palparse las puntas de los implantes en la región ventral o lateral del pene, a menudo no colocadas dentro del glande. Esta complicación puede generar dolor en el sitio de la extrusión o dolor referido por la pareja durante el coito. ${ }^{10,11}$

La extrusión se produce cuando la túnica albugínea distal se debilita por uno o más de los siguientes factores: dilatación agresiva, colocación de un cilindro en una cavidad corporal demasiado estrecha o por presión mecánica del cilindro. ${ }^{12}$ Es importante reconocer estas complicaciones y estar familiarizado con las técnicas quirúrgicas para efectuar el procedimiento adecuado.

\section{CASO CLÍNICO}

Paciente masculino de 65 años de edad, con antecedente de colocación de prótesis peneana de 2 componentes en 2008; posteriormente tuvo disfunción mecánica, que ameritó el recambio de la prótesis por una de 3 componentes en agosto de 2016.

El paciente inició su padecimiento actual en marzo de 2017, con dolor en la región distallateral derecha del cuerpo del pene. Los estudios de laboratorio reportaron: hemoglobina 13.7 $\mathrm{g} / \mathrm{dL}$, leucocitos $6.8 / \mathrm{mL}$, plaquetas $300 / \mathrm{mm}^{3}$ y creatinina $0.4 \mathrm{mg} / \mathrm{dL}$. El examen general de orina y urocultivo resultaron negativos para infección. 
A la exploración física se encontró: tensión arterial $130-80 \mathrm{mmHg}$ frecuencia cardiaca 72 Ipm y frecuencia respiratoria $16 \mathrm{rpm}$. A la palpación: abultamiento e induración con prótesis inactivada.

La extrusión protésica se confirmó con resonancia magnética de alta resolución espacial en T2, con la prótesis en reposo y activada; no había perforación de la túnica albugínea (Figura 1).

De acuerdo con los hallazgos el procedimiento quirúrgico se efectuó con la técnica de Mulcahy (representa una técnica sencilla y durante el mismo procedimiento puede realizarse el recambio y no se utiliza ningún material de

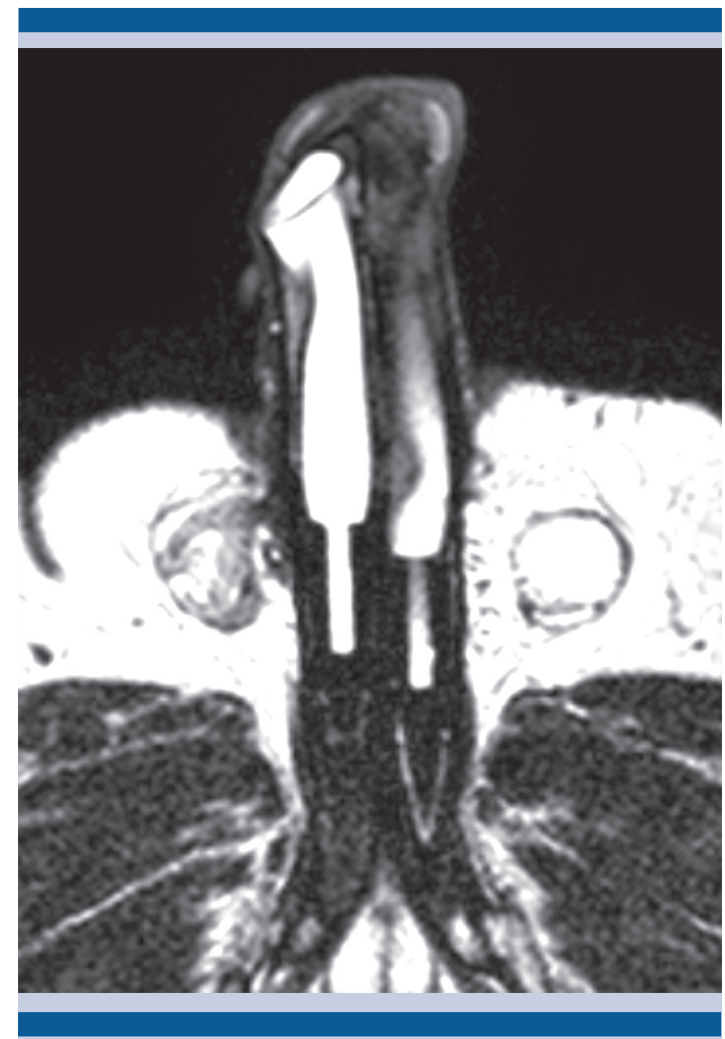

Figura 1. Resonancia magnética que evidencia la prótesis inactiva en estado flácido y la angulación del cilindro derecho por la falta de rigidez de la túnica albugínea. injerto sintético). Con esta técnica se realiza el recambio protésico completo, con la extracción de todos sus componentes y colocación de la nueva prótesis de 3 componentes $\left(\operatorname{Titan}^{\circledR}\right)$.

El procedimiento se efectuó con corporotomía distal e incisión capsular transversal de la corpora en posición dorsal, además de una nueva dilatación en la porción distal, y se colocó la prótesis en el nuevo trayecto (pseudocápsula). La prótesis peneana se activó a las 6 semanas posquirúrgicas, con evolución funcional satisfactoria y sin percepción de dolor por el paciente. La resonancia magnética de control mostró la prótesis en adecuada posición (Figura 2).

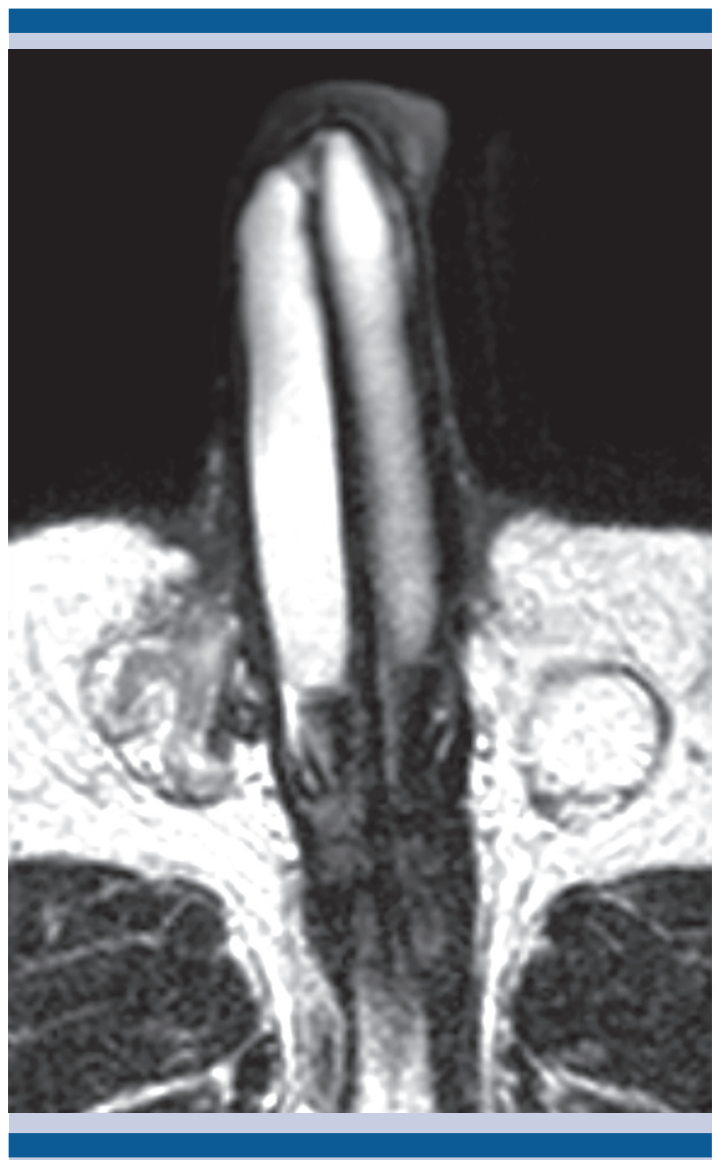

Figura 2. Resonancia magnética que muestra la prótesis peneana activada después de 6 semanas posquirúrgicas, con evolución funcional satisfactoria. 


\section{DISCUSIÓN}

La primera prótesis para conferir rigidez al pene la produjo Boronas en 1936, quien utilizó cartílago costal autólogo dentro de un flap dérmico tubularizado, con la finalidad de reconstruir un pene amputado de forma traumática. ${ }^{16}$ Bergmann publicó una técnica similar, en la que el cartílago se implantaba entre los remanentes de los cuerpos cavernosos amputados. ${ }^{13}$

En 1952, Goodwin y Scott reportaron los primeros implantes aloplásticos en la reconstrucción del pene después de una amputación, en un paciente a quien le implantaron prótesis acrílicas, colocadas fuera de los cuerpos cavernosos, debajo de la fascia de Buck.

En 1966, Beheri fue el primero en utilizar 2 cilindros de polietileno colocados en el interior de los cuerpos cavernosos. La descripción de la técnica no ha variado sustancialmente de la que se utiliza en la actualidad.

En 1973 Scott desarrolló las prótesis de pene con mecanismo hidráulico, lo que supuso el último gran avance en la cirugía protésica peneana. ${ }^{14}$

La túnica albugínea que se encuentra en la porción distal del cuerpo del pene es más delgada que la situada en su base, particularmente en el aspecto ventral, donde la mayor parte de las prótesis tiende a extruirse; por tanto, esto sugiere una base anatómica para tal proceso. Otras posibles causas de extrusión incluyen: utilizar cilindros de mayor dimensión y realizar una dilatación distal excesivamente vigorosa; provocar microperforaciones durante la dilatación de los cuerpos cavernosos distales con dilatadores de Hegar de pequeño calibre (6 a 8 Fr) o por infección. Las prótesis maleables se han asociado con mayor porcentaje de erosión debido a la presión constante que ejercen; sin embargo, diversos estudios no reportan si este tipo de prótesis pe- neanas generan mayor tasa de complicaciones que las prótesis de 3 componentes. ${ }^{15}$ Las tasas de extrusión y erosión se han notificado en 1.2-8\% de las prótesis inflables.

La erosión del implante distal es un resultado desafortunado, pues requiere el retiro de todo el dispositivo. El material del implante expuesto hace que el dispositivo se infecte, incluso en ausencia de síntomas clínicos. En estos casos es importante resolver la extrusión de forma lateral, antes de que se produzca la erosión.

El cirujano debe decidir reemplazar o no todo el dispositivo. Diversos estudios sugieren que puede utilizarse el mismo dispositivo si tiene menos de 2 años de haberse colocado; la mayoría de los urólogos utiliza un dispositivo nuevo cuando la prótesis peneana inflable tiene más de 5 años de haber sido colocada. Actualmente no existe información suficiente que respalde este hecho. En caso de utilizar el mismo dispositivo se recomienda realizar una incisión distal en el sitio de la extrusión, pero si se reemplaza el dispositivo entero, entonces se requiere una incisión distal y penoescrotal para el retiro de la prótesis inflable antigua y la colocación del nuevo implante peneano.

La resonancia magnética constituye el método de elección para la valoración preoperatoria en pacientes con antecedente de prótesis peneana o para el estudio de las complicaciones posquirúrgicas, pues identifica los componentes protésicos o abscesos con una sensibilidad diagnóstica de $95.8 \%$. Se utilizan antenas de superficie multicanal y secuencias de alta resolución espacial potenciadas en T2 y en los tres planos (axial, coronal y sagital). En la primera fase del estudio se coloca el pene en posición anatómica extendido sobre el abdomen y en estado de flacidez. La segunda fase se realiza en estado de erección, con la prótesis peneana activada. ${ }^{16}$ 
Después de la extracción de la prótesis peneana se genera un proceso inflamatorio importante, con posterior fibrosis y cicatrización de los cuerpos cavernosos, que casi siempre resulta en acortamiento del pene y dificultad para reinsertar un nuevo dispositivo, incluso es casi imposible.

Para evitar este tipo de sucesos existen múltiples técnicas para el tratamiento de la extrusión de prótesis peneana, según su localización, tipo (perforación o no de la uretra) y coexistencia o no de algún proceso infeccioso, con la finalidad de establecer el tratamiento adecuado. Otra opción de rescate de las prótesis infectadas incluye el uso de drenajes cerrados e irrigación, con indicación de diversos tipos de antibióticos, cuyo objetivo es disminuir la cicatrización o realizar un reemplazo temprano (antes de 72 horas) con un nuevo dispositivo o el rescate inmediato y el reemplazo de la prótesis peneana con la técnica de Mulcahy, donde se utiliza la cápsula que se forma en el recambio protésico y representa una alternativa con resultado satisfactorio.

Smith y su grupo describieron el uso de politetrafluoretileno (GoreTex) en el sitio de la erosión. El riesgo de infección es mayor cuando se utiliza material de injerto sintético. Este tipo de intervención implica mayor tiempo quirúrgico, mayor cantidad de material no absorbible y de cuerpos extraños en el pene, y mayor número de incisiones. ${ }^{17}$

La técnica de Alter es una opción adicional, en la que se utiliza una aleta prefabricada de túnica vaginal para reconstruir la túnica albugínea en el sitio de extrusión de la prótesis. El método comprende dos etapas: en la primera, la fascia del músculo recto abdominal se traslada a la túnica vaginal del testículo y en la segunda, dos semanas más tarde, el colgajo prefabricado de la túnica vaginal se moviliza a la corpora distal, entre el cilindro y la fina capa de la túnica albugínea (para la extrusión lateral de la túnica), con la finalidad de reemplazar una porción de la túnica albugínea debilitada y sirva como reIleno de tejido entre el cilindro y la uretra (para la extrusión de la túnica medial). Esta técnica se reserva para pacientes con antecedente de dos o más extrusiones de prótesis peneana.

La técnica implementada en el paciente de este estudio fue, inicialmente, la descrita por Mulcahy (1996): se eliminaron todos los componentes del dispositivo, se limpiaron los espacios de los componentes con diversas soluciones antisépticas y se colocó el nuevo implante en el mismo sitio.

El tratamiento prequirúrgico y la técnica de Mulcahy se realiza de la siguiente manera:

Antes de la cirugía se sugiere evaluar la coexistencia de fibrosis de los cuerpos cavernosos, cicatrices retropúbicas o enfermedad de Peyronie. En caso de reemplazo de la prótesis peneana debe determinarse si la túnica albugínea está intacta y se encuentran datos de erosión inminente.

Se ha demostrado que el lavado, durante la revisión quirúrgica, reduce la tasa de infección en pacientes con prótesis inflables, clínicamente no infectados. ${ }^{18}$ Antes de la intervención quirúrgica se afeita el área abdomino-genital y posteriormente se efectúa la limpieza del área quirúrgica con isodine (espuma).

Mientras que el protocolo tradicional de rescate descrito por Mulcahy utiliza múltiples soluciones y cambio de batas, guantes, sábanas quirúrgicas e instrumentos, el lavado de revisión suele hacerse con tres o cuatro jeringas asepto, con irrigación de cada espacio del implante. ${ }^{19}$

Se coloca una sonda Foley para delimitar y proteger la uretra lo mejor posible. Se realiza corporotomía distal para el tratamiento de la extrusión lateral de los cilindros de la prótesis peneana y se utiliza el propio tejido del paciente, 
con una cápsula fibrosa que rodea la prótesis inflable. Se efectúa una incisión transversal, de aproximadamente $4 \mathrm{~cm}$, a 1 o $2 \mathrm{~cm}$ proximal de la punta del cilindro que se está extruyendo; posteriormente se diseca en dirección proximal, a través de la túnica albugínea, con cuidado de no dañar el cilindro de la prótesis inflable, en caso de tener planeado utilizar el mismo implante.

Se tracciona la punta del cilindro a través de la incisión y se retrae la prótesis peneana proximalmente, con la finalidad de exponer la superficie medial del espacio del cilindro (Figura 3). A continuación se efectúa una incisión transversal en la pared medial del espacio del cilindro, mediante el tejido eréctil, para formar una pseudocápsula; el espacio recién formado se dilata apropiadamente para que coincida con el calibre de la prótesis y ésta se pasa a través de dicha pseudocápsula.
Enseguida se sutura la porción medial de la pseudocápsula, en la pared lateral de la cápsula, en su porción distal, cerrando efectivamente el espacio de la prótesis previa o extruida, y se crea una doble capa de tejido (cápsula original) para proteger la porción distal del cilindro.

Con la aguja de Keith y el introductor de Furlow se redirige el cilindro y se coloca en la nueva cavidad (Figura 4). Se realiza el cierre de la túnica albugínea con sutura absorbible para reforzar la reparación y evitar lesionar el cilindro. En caso de utilizar un injerto de parche, este es el momento adecuado. La pseudocápsula es lo suficientemente resistente para proteger de posibles erosiones.

De manera similar, para la inminente erosión medial de la punta distal del cilindro, típicamente
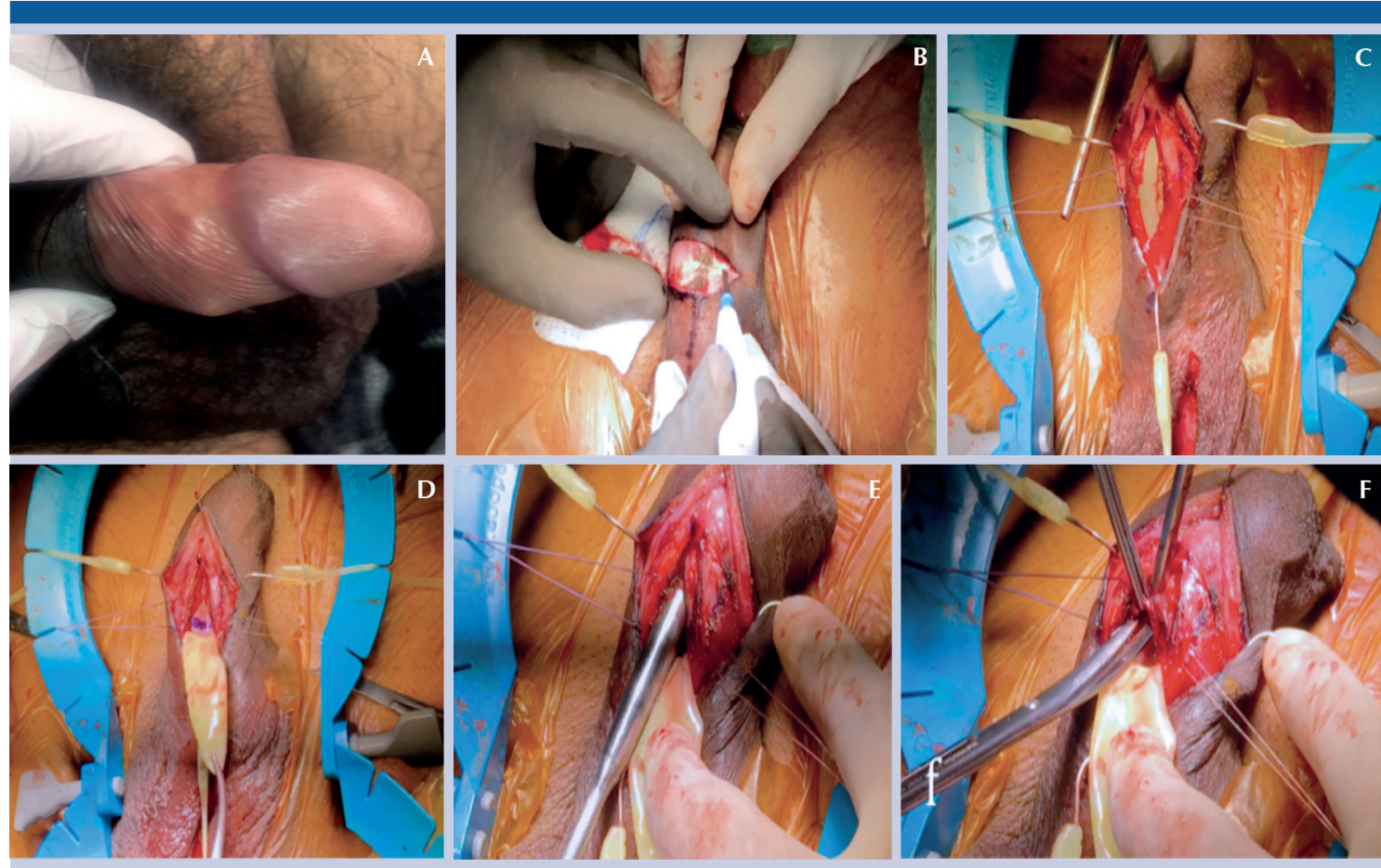

Figura 3. Extrusión en la cara lateral derecha del cuerpo del pene (A); incisión externa transversal y longitudinal en los cuerpos cavernosos (B Y C); extracción de la prótesis extruida (D); nueva dilatación sobre el cuerpo cavernoso derecho (E Y F). 


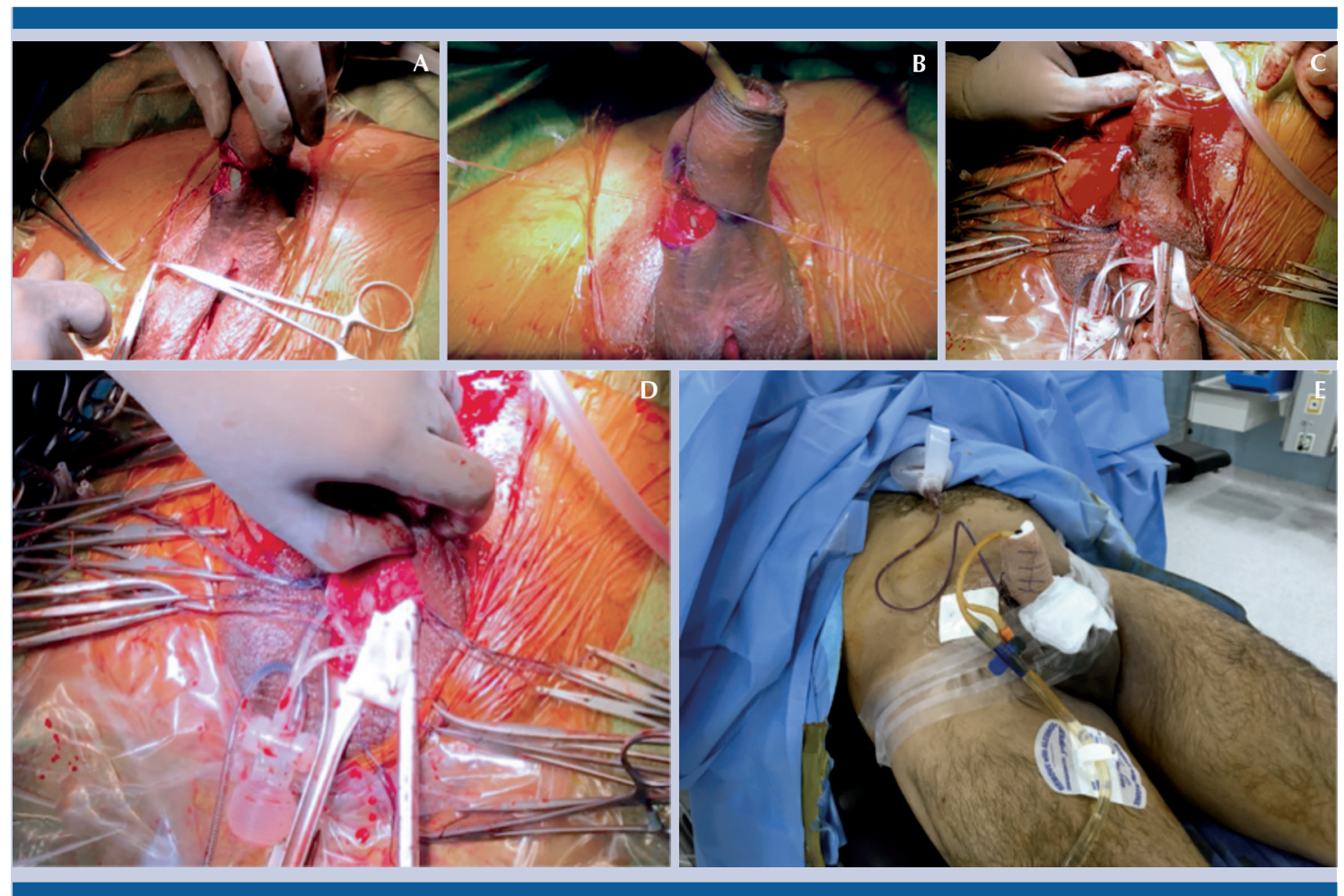

Figura 4. Reposición de la prótesis de manera distal (A), cierre de la túnica albugínea (B); reposición de la porción proximal de ambas prótesis (C Y D); permanencia de drenaje a succión, derivación urinaria y compresión del sitio quirúrgico $(\mathbf{E})$.

ubicada en la fosa navicular, se repara o coloca el injerto tisular natural con el "nuevo" canal dilatado, sólo lateral a la pared de la cápsula. La reparación de la erosión medial suele ser más fácil que la de la extrusión lateral, pues la uretra se encuentra en menor riesgo de lesionarse.

La colocación de prótesis peneanas es un procedimiento poco común en la población mexicana; por tanto, existen pocos casos reportados en la bibliografía y la experiencia en la resolución de extrusiones es escasa. La técnica de Mulcahy representa una opción efectiva, con alta tasa de éxito y baja de infección en pacientes con extrusión de prótesis en la porción lateral del pene.

\section{REFERENCIAS}

1. Mulcahy J, Austoni E, Barada JH, Hellstrom WJ, et al. The penile implant for erectile dysfunction. J Sex Med 2004;1:98-109.
2. Trost L, Hellstrom WJ. History, contemporary outcomes, and future of penile prostheses: A review of the literature. Sex Med Rev 2013;1(3):150-163.

3. Hellstrom WJ, Montague DK, Moncada I, Carson C, et al. Implants, mechanical devices, and vascular surgery for erectile dysfunction. J Sex Med 2010;7:501-523.

4. Martinez-Salamanca JI, Mueller A, Moncada I, Carballido J, Mulhall JP. Penile prosthesis surgery in patients with corporal fibrosis: a state of the art review. J Sex Med 2011;8(7):1880-9.

5. Montague DK. Penile prosthesis implantation in the era of medical treatment for erectile dysfunction. Urol Clin North Am 2011;38:217.

6. Dhabuwala C, Sheth S, Zamzow B. Infection rates of rifampin/gentamicin-coated Titan Coloplast penile implants. Comparison with Inhibizone-impregnated AMS penile implants. J Sex Med 2011;8(1):315-20.

7. Hinds PR, Wilson SK, Sadeghi-Nejad H. Dilemmas of inflatable penile prosthesis revision surgery: What practices achieve the best outcomes and the lowest infection rates? (CME). J Sex Med 2012;9:2483-92

8. Henry GD, Kansal NS, Callaway M, Grigsby T, et al. Centers of excellence concept and penile prostheses: An outcome analysis. J Urol 2009;181:1264-8. 
9. Vitarelli A, Divenuto L, Fortunato F. Long term patient satisfaction and quality of life with AMS700CX inflatable penile prosthesis. Arch Ital Urol Androl 2013;85(3):133-7.

10. Mulcahy JJ. The prevention and management of noninfectious complications of penile implants. Sex Med Rev 2015;3:203-13

11. Mulcahy JJ. Distal corporoplasty for lateral extrusion of penile prosthesis cylinders. J Urol 1999;161:193-5.

12. Trost LW, Baum N, Hellstrom WJ. Managing the difficult penile prosthesis patient. J Sex Med 2013;4:893-906.

13. Bergmann RT, Howard A, Barnes RW. Plastic reconstruction of the penis. J Urol 1948;59(6):1174-86.

14. Kiliçarslan H, Kaynak Y, Gökcen K, et al. Comparison of patient satisfaction rates for the malleable and two pieceinflatable penile prostheses. Turk J Urol 2014;40(4):207-10.
15. Shaeer O. Management of distal extrusion of penile prosthesis: partial disassembly and tip reinforcement by double breasting or grafting. J Sex Med 2008;5(5):1257-1262.

16. Pacheco Usmayo A, et al. Utilidad de la resonancia magnética en la valoración postquirúrgica de pacientes con prótesis hidráulica de pene. Radiologia 2017;59(6):504-510.

17. Smith C, Kraus S, Boone T. Management of impending penile prosthesis erosion with a polytetrafluoroethylene distal wind sock graft. J Urol 1998;160(6 Pt 1):2037-40.

18. Villarreal $\mathrm{H}$, Jones $\mathrm{L}$. Outcomes of and satisfaction with the inflatable penile prosthesis in the elderly male. Adv Urol 2012;2012:240963.

19. Nehra A, Carson C, Chapin AK. Long-term infection outcomes of 3-piece antibiotic impregnated penile prostheses used in replacement implant surgery. J Urol 2012;188(3):899-903.

\section{AVISO IMPORTANTE}

La Revista Mexicana de Urología se convierte en una publicación solo digital, con todas las ventajas que los medios y dispositivos electrónicos ofrecen. Usted podrá revisar la información mediante el sitio web (www.revistamexicanadeurologia.org.mx) o descargando la app para Android o iPhone.

Para consultar el texto completo de los artículos deberá registrarse por una sola vez con su correo electrónico, crear una contraseña, indicar su nombre, apellidos y especialidad.

Esta información es indispensable para saber qué consulta y cuáles son sus intereses, y poder en el futuro inmediato satisfacer sus necesidades de información.

App Store 